basing his theory on Nachtsheim's figures, considers that the male and female honey bee are somatically diploid and tetraploid respectively, the haploid number being 8 . Nachtsheim's results do not agree with those of Petrunkewitsch, who found 16 chromosomes in the oocyte, nor of Meves, who demonstrated 16 in the second spermatocytes, nor with our findings, especially those relating to oogonia and egg maturation. Furthermore, Nachtsheim's results have long puzzled cytologists, but since our new evidence clarifies the situation, Whiting's views on polyploidy in the bee appear inacceptable.

Our conclusions have been reached after a study of a wealth of material which permits us to stress the following points on honey bee cytology: (1) the well-defined shapes and individuality of the chromosomes; (2) their discrete arrangement at all times; (3) the haploid-diploid constitution of the male and female, the male having 16 and the female 32 chromosomes in gonial and in normal somatic cells; (4) the invalidity of Nachtsheim's evidence regarding "Sammelchromosomen"; and (5) the inacceptability of Whiting's theory on somatic polyploidy in the honey bee.

We wish to acknowledge indebtedness to Mr. D. Robb for the use of an observation hive, to Mr. D. Gauld, and to many other local beekeepers for their ready co-operation in supplying suitable material, and to Mr. H. R. Ness for invaluable help with the photography. Prof. A. D. Peacock, in whose department this work has been carried on, has throughout given helpful advice and criticism.

'Petrunkewitsch, A., Zool. Jahrb., 14 (1901).

2 Petrunkewitsch, A., Zool. Jahrb., 17 (1903).

${ }^{3}$ Doncaster, L., Anat. Anz., 29 (1906).

'Doncaster, L., Anat. Anz., 31 (1907).

'Mark, E. L., and Copeland, M., Proc. Amer. Acad. Arts Sci., 42 (1906).

'Meves, F., Arch. Mikr. Anat, 70 (1907).

Nachtsheim, H., Sitzungber. Ges. Morph. Phys. Munich (1912).

Nachtsheim, H., Archiv. f. Zellforsch., 11 (1913).

- Jegen, J., Z. f. Zschokke, Basel (1920).

"Sanderson, A. R., Genetica, 14 (1932).

"Whiting, A. R., Quart. Rev. Biol., 20 (1945).

12 Granata, L., Biologica, 2 (1909).

${ }^{13}$ Granata, L., Monit. Zool. Ital., Anno 24 (1913).

\section{VENEREAL DISEASE CONTROL IN POLAND}

$\mathrm{P}_{\mathrm{p}}^{\mathrm{o}}$ LAND is at present faced with a venereal disease problem against which the state of affairs in Britain appears insignificant. The incidence of venereal diseases has increased enormously over the war years and the number of fresh infections with syphilis is now calculated to be about 150,000 per year. There is a decrease in live births of about $40,000-50,000$ per year, the mortality-rate from syphilis being about 11 per cent. The reason for this state of affairs is not far to seek. The Germans spared little attention for the Poles whether they were in Poland or displaced to other countries. Venereal diseases were prevalent among displaced persons, and although plans were formulated before D-day for the treatment of infected persons before they were repatriated, nothing was, in fact, ever done for them.

The Ministry of Health in Warsaw has now prepared a permanent programme for venereal diseases control and a temporary programme for immediate operations. The basic units are consultation centres for skin and venereal diseases in health centres distributed throughout the country, and there are, of course, clinics in the hospitals of the major cities.

The basis is a network of medical facilities free to all. The principle of the legal basis is that every citizen with venereal disease can, if necessary, be compelled to submit to treatment. Sources of infection are to be discovered and put under treatment. Auxiliary measures include periodic surveys of persons susceptible to infection; obligatory pre-marital examination; obligatory serum tests for syphilis in pregnant women; training of medical personnel ; propaganda; control of prostitution and vagrancy; control of alcoholism; and collection of statistics. Every physician, regardless of his status, is required to determine, and report to the nearest centre, all the sexual contacts of his patients during the infectious period. Persons so named are requested to attend the centre within seven days, and if there is no response a health visitor is to be sent. If this is still unavailing, compulsion is used. Mobile ambulance units are to be used in isolated districts for treatment, propaganda, mass observation, training, etc. Training courses, to be attended at least once a year by the medical men in centres, are to be held in the main provincial centres and will include diagnostic methods, modern treatment and management of complications, control and organisation, and propaganda organisation. Propaganda will consist in popular lectures, films and slides, posters and pamphlets and anti-alcohol appeals.

This programme is directed to the rapid treatment of syphilis during 1948. The old long-term treatment is discarded in early syphilis because of the high rate of default and possibility of continuing infectiousness. Use of penicillin reduces treatment to about two weeks and the infectious stage to about the same period; but there is a risk of infectious relapse in 15 per cent of cases.

It is proposed to do sample surveys of fifteen groups of a thousand people in various areas to gain information on prevalence and distribution of disease.

The importance of accurate serological examina. tions is recognized and a standard (flocculation) test is to be used throughout the country. Private laboratories are to be checked each year to ensure efficiency. Serological examination is to be routine on first diagnos's of pregnancy, and for military recruits, students and workers.

In the rapid treatment scheme every doctor, in public or in private practice, is to be employed. High standards in diagnostic technique are required.

Rapid treatment is to be given to all cases of symptomatic early syphilis and to cases of asymptomatic early syphilis (up to three years from date of infection) which have not previously been treated or which have received less than one standard course of arsenic and bismuth. The treatment is to consist of $3,000,000$ units of penicillin in oil-beeswax suspension given in doses of 300,000 units each day for ten days. $0.2 \mathrm{gm}$. bismuth subsalicylate is to be given on the first, fourth, seventh and tenth days of treatment. The bismuth is omitted in pregnant women. Children are to receive 200,000 units penicillin per kilo body weight.

Intensive propaganda on the necessity for early treatment and systematic follow-up and on the availability of free treatment facilities is to be carried on by posters, newspaper articles, radio and films. 
The rapid treatment method is only intended for use in the early stages of syphilis, early cases of latent syphilis, in pregnant women at any stage of disease, and in congenital syphilis, inadequately treated, up to the third year. Diagnosis is to depend on the finding of Treponema Pallidum by darkground examination, a positive serum test in the presence of clinical signs, or two positive serum tests in the absence of clinical signs if the history suggests an infection of less than three years duration.

Drugs are always available at centres; but a private practitioner receives supplies only after notifying a case and sending a requisition form. This will inevitably lead to delay in starting treat. ment in many cases. Private practitioners may charge a fee for treatment, but have to inform the patient that the drugs are supplied free.

Follow-up after treatment consists in clinical and serological examinations once a month for the first six months, every three months in the second six months, and each six months in the second year. The cerebro-spinal fluid is examined six months and two years after treatment. Failures after rapid treatment are to receive four courses of arsenic and bismuth on the long-term plan.

The examining physician must obtain from the patient particulars of all his contacts from the time of contracting the disease (eight weeks before the first sign in the case of syphilis) until the time of diagnosis. The patient is asked to try to bring for examination all such contacts; but methods of compulsion can be used as a last resort if persuasion is unsuccessful.

The theoretical basis of this plan is excellent, embodying as it does the latest features of AngloAmerican practice. It is complicated in practice by the necessity for a formidable amount of documentation on the patient, treatment, drugs, follow-up, contacts, statistics, etc. Indeed, the paper-work will consume more man-hours than diagnosis and treatment, as was the case in the Services during the War.

The choice of treatment schedule has been influenced, no doubt, by considerations of time and availability of drugs. I do not consider, personally, that the addition of $0.8 \mathrm{gm}$. bismuth subsalicylate will increase the 'cure' rate. I believe it will do no more than delay the appearance of signs of relapse in cases which will fail. There is nothing to show that bismuth and penicillin are synergistic in action. The effect of penicillin during a rapid treatment would be more likely to be enhanced by the addition of arsenic (as an arsenoxide for preference) to the schedule. This idea has, no doubt, been discarded because of the slight risk of arsenical toxic effects and because of its technical difficulties. Arsenicals have to be administered intravenously, and it is probable that Poland at the present time lacks the skilled personnel necessary to cope with these added difficulties in the vast numbers of cases to be treated.

The choice of $3,000,000$ units as the total dose of penicillin is again, no doubt, governed by circum. stances ; but it is a minimal effective dose and ought to be doubled as soon as supplies are available. With an infectious relapse-rate estimated (conservatively in my opinion) at 15 per cent, the follow-up programme must be very carefully carried out and failures re-treated without delay if an end state worse than the original is not to follow an initial apparent success.
The Polish medical authorities are to be congratulated on a carefully prepared and ambitious plan which seems to cover the immediate needs and make a basis for a useful service in the future. The statistical service may prove of great value in the rapid assessing of any treatment schedule, and it is to be hoped that if it is a success the system will be used for comparative experiments in treatment.

James Marshall

\section{DECARBOXYLATION OF OXALACETATE BY BLOOD}

\author{
BY PETER M. NOSSAL
}

Department of Biochemistry, University of Adelaide

GVOLUTION of carbon dioxide from oxalacetate 1. by washed rabbit erythrocytes at $p \mathbf{H} 7 \cdot 4$ has been reported previously ${ }^{1}$. Because of the solubility of carbon dioxide at that $p H$, this could at best be only a qualitative indication of the decarboxylation. Working in either phosphate or $2 M$ acetate buffer, and measuring carbon dioxide formation by the standard Warburg technique, the process has been thoroughly investigated.

The process is found in the following constituents of rabbit blood, either freshly drawn or stored for periods (up to several days) on ice: whole blood, erythrocytes, erythrocyte hæmolysate, plasma and acetone powder extracts of all the aforementioned. Hæmolysates were usually the most active, whole blood, erythrocytes and plasma roughly equally active, and acetone powder extracts all slightly less active.

At $p H 6 \cdot 5$, no decarboxylation of either $\alpha$-keto. glutarate or pyruvate was observed, whereas oxalacetate decarboxylation was considerable; thus the system seems to be specific for that substrate.

Dialysis for 20-24 hours against distilled water caused reduction in activity. Whereas neither man. ganous ions $\left(\mathrm{Mn}^{++}\right)$nor magnesium ions $\left(\mathrm{Mg}^{++}\right)$had any influence on the decarboxylation by any of the above preparations prior to dialysis, partial restoration of activity in dialysed extracts was observed by fairly high concentrations $\left(10^{-3} M-10^{-2} M\right)$ of manganous ions, and, less markedly, magnesium ions. Thiamine does not feature in the process under the experimental conditions employed. Added pyruvate in no way affects carbon dioxide output, so that the reaction does not seem to be a Krebs condensation to cis. aconitate. The process is not markedly reduced by submersion of the enzyme in water baths at $70-75^{\circ} \mathrm{C}$. for periods of 1-4 min.

It must be noted that oxalacetate itself undergoes a quite rapid autodecomposition under the experi. mental conditions. This is very sensitive to $p \mathbf{H}$ and varies somewhat for each different oxalacetate solu. tion, so that all experiments were invariably run with duplicate blanks containing only the appropriate buffer and the substrate. Influence of $p . \mathbf{H}$ on auto. decomposition of oxalacetate is shown below, that at $p H \mathbf{H}$ being taken as 100 for comparative purposes: $\begin{array}{lllllllll}p \mathrm{H} & 3 \cdot 5 & 4 \cdot 0 & \mathbf{4} \cdot 5 & 5 \cdot 0 & 5 \cdot 5 & 6 \cdot 0 & 6 \cdot 5 & 7 \cdot 0 \\ \text { Decarboxylation } & 300 & \mathbf{2 8 0} & \mathbf{2 5 0} & \mathbf{1 8 0} & \mathbf{1 5 0} & 150 & 130 & 100\end{array}$

All references made to decarboxylations are to be taken as increases above the autodecomposition rate, obtained after duly correcting for autodecomposition blanks. 